\title{
Unicorn paths and hyperfiniteness for the mapping class group
}

\author{
Piotr Przytycki and Marcin Sabok
}

Department of Mathematics and Statistics, McGill University, Burnside Hall, 805 Sherbrooke St. W, Montreal, Quebec, Canada H3A 0B9; E-mail: piotr.przytycki@mcgill.ca; marcin.sabok@mcgill.ca.

Received: 31 July 2020; Revised: 16 December 2020; Accepted: 22 January 2021

2020 Mathematics Subject Classification: 57K20, 37A20, 03E15

\begin{abstract}
Let $S$ be an orientable surface of finite type. Using Pho-on's infinite unicorn paths, we prove the hyperfiniteness of orbit equivalence relations induced by the actions of the mapping class group of $S$ on the Gromov boundaries of the arc graph and the curve graph of $S$. In the curve graph case, this strengthens the results of Hamenstädt and Kida that this action is universally amenable and that the mapping class group of $S$ is exact.
\end{abstract}

\section{Introduction}

An equivalence relation $E$ on a standard Borel space $X$ is Borel if $E$ is a Borel subset of $X \times X$. An equivalence relation is countable (resp., finite) if every equivalence class is countable (resp., finite). Given a Borel action of a countable group on a standard Borel space $X$, the induced orbit equivalence relation is a countable Borel equivalence relation. A Borel equivalence relation $E$ is hyperfinite if $E$ can be written as an increasing union of a sequence of finite Borel equivalence relations.

Let $S$ be an oriented surface of genus $g \geq 0$ with $n \geq 0$ punctures, of negative Euler characteristic. We denote by $\mathcal{A}(S)$ (for $n \geq 1$ ) and $\mathcal{C}(S)$ its arc graph and its curve graph, respectively, which are Gromov hyperbolic (see Section 2). The actions of the mapping class group $\operatorname{Mod}(S)$ on $\mathcal{A}(S)$ and $\mathrm{C}(S)$ by automorphisms extend to actions on their Gromov boundaries by homeomorphisms. Our main result is the following:

Theorem 1.1. The orbit equivalence relation on $\partial \mathcal{A}(S)$ induced by the action of $\operatorname{Mod}(S)$ is hyperfinite.

As a consequence we will derive the following:

Corollary 1.2. The orbit equivalence relation on $\partial \mathcal{C}(S)$ induced by the action of $\operatorname{Mod}(S)$ is hyperfinite.

This strengthens the results of Hamenstädt [Ham09, Cor 2] and Kida [Kid08, Thm 1.4(ii)] that this equivalence relation is universally amenable (for a definition, see Section 1.1).

We will also obtain the following (for the definition of $\mathcal{C} \mathcal{L}(S)$, see Section 5):

Corollary 1.3. The orbit equivalence relation on the space of complete geodesic laminations $\mathcal{C} \mathcal{L}(S)$ induced by the action of $\operatorname{Mod}(S)$ is hyperfinite.

In particular, this equivalence relation is universally amenable. Since $\mathcal{C} \mathcal{L}(S)$ is compact and Hausdorff, and its point stabilisers are virtually abelian, this gives a new proof of [Ham09, Thm 1] that the

(C) The Author(s), 2021. Published by Cambridge University Press. This is an Open Access article, distributed under the terms of the Creative Commons Attribution licence (http://creativecommons.org/licenses/by/4.0/), which permits unrestricted re-use, distribution, and reproduction in any medium, provided the original work is properly cited. 
action of $\operatorname{Mod}(S)$ on $\mathcal{E} \mathcal{L}(S)$ is topologically amenable (see Section 1.1). This implies that $\operatorname{Mod}(S)$ is exact (which was proved independently to Hamenstädt by Kida [Kid08, Thm C.5]).

\subsection{Amenability}

Let $\mu$ be a Borel probability measure on a standard Borel space $X$. The notion of amenability for a measurable action of a countable group on $(X, \mu)$ was introduced by Zimmer [Zim78] (see also [Zim84]) and has many equivalent definitions (see, e.g., [AEG94]; for a more general definition for measured groupoids, see [ADR00]). It is closely related to the notion of amenability for countable Borel equivalence relations [Gao09, Def 7.4.5] (see also [Kec93, §3] or [Moo20, §3]). Namely, a measurable action on $(X, \mu)$ is $\mu$-amenable if and only if $\mu$-almost all stabilisers are amenable and the induced orbit equivalence relation on $X$ is $\mu$-amenable [AEG94, Thm 5.1]. A countable Borel equivalence relation on $X$ or a Borel action of a countable group on $X$ is universally amenable if it is $\mu$-amenable for every quasiinvariant Borel probability measure $\mu$ on $X$ (or, equivalently, for every Borel probability measure $\mu$ on $X$ [KM04, Cor 10.2]). In particular, any Borel action of an amenable group is universally amenable.

By [ADR00, Thm 3.3.7], an action of a countable group $G$ by homeomorphisms on a locally compact Hausdorff space $X$ is universally amenable if and only if it is topologically amenable (for a definition, see [Oza06b, Def 2.1]). If $X$ is compact Hausdorff, then the topological amenability of the action implies the exactness of $G$ [AD02, Thm 7.2].

Boundary actions have been studied extensively from the point of view of amenability. Connes, Feldman and Weiss [CFW81, Cor 13] and, independently, Vershik [Ver78, Thm 2], showed that the tail equivalence relation discussed in Section 1.2 is universally amenable, which implies that the induced action of the finitely generated free group $F_{n}$ on its Gromov boundary $\partial F_{n}$ is universally amenable. This was later generalised by Adams [Ada94] to all hyperbolic groups (see also [Kai04]). Furthermore, Ozawa [Oza06a] proved that the action of a relatively hyperbolic group with amenable parabolic subgroups on the Gromov boundary of its coned-off Cayley graph is topologically amenable. Moreover, Nevo and Sageev proved that the action of a cocompactly cubulated group on a particular subset of its Roller boundary is universally amenable [NS13]. Lécureux proved that if a group $G$ acts geometrically on a building $X$, then the action of $G$ on the combinatorial boundary of $X$ is topologically amenable [Léc10]. Finally, Bestvina, Guirardel and Horbez proved that the action of $\operatorname{Out}\left(F_{n}\right)$ on the Gromov boundary of its free factor complex is universally amenable (see [BGH21, Thm 6.4] and [GHL20, Prop 7.2]), which uses the description of the Gromov boundary of the free factor complex in [BR15] and [Ham14].

\subsection{Hyperfiniteness}

As shown independently by Weiss and Slaman-Steel [Gao09, Thm 7.2.4], a Borel equivalence relation $E$ is hyperfinite if and only if there is a Borel action of $\mathbb{Z}$ inducing $E$ as its orbit equivalence relation. Since $\mathbb{Z}$ is amenable, the hyperfiniteness of a Borel equivalence relation implies its universal amenability. It is a well-known open problem whether the converse holds - that is, whether a universally amenable Borel equivalence relation is always hyperfinite. Connes, Feldman and Weiss [CFW81, Thm 10] (see also [KM04, Thm 10.1]) showed that a $\mu$-amenable Borel equivalence relation on $X$ becomes hyperfinite after removing from $X$ a set of $\mu$-measure 0 .

The relative complexity of Borel equivalence relations is measured by Borel reducibility. Given two equivalence relations $E$ and $F$ on standard Borel spaces $X$ and $Y$, respectively, a function $f: X \rightarrow Y$ is a Borel reduction from $E$ to $F$ if $f$ is a Borel function and for every $a, b \in X$ we have $a \sim_{E} b$ if and only if $f(a) \sim_{F} f(b)$. A relation $E$ is Borel reducible to $F$ if there exists a Borel reduction from $E$ to $F$. The relation $E_{0}$ is defined on $\{0,1\}^{\mathbb{N}}$ (with the product topology) as $\left(a_{i}\right)_{i=0}^{\infty} \sim E_{0}\left(b_{i}\right)_{i=0}^{\infty}$ if $a_{i}=b_{i}$ for all $i$ sufficiently large. It is easy to see that $E_{0}$ is hyperfinite. In fact, a countable Borel equivalence relation is hyperfinite if and only if it is Borel reducible to $E_{0}$ [Gao09, Thm 7.2.2].

Let $\Omega$ be a countable set with discrete topology. The tail equivalence relation $E_{t}$ on $\Omega^{\mathbb{N}}$ is defined as $\left(a_{i}\right)_{i=0}^{\infty} \sim_{E_{t}}\left(b_{i}\right)_{i=0}^{\infty}$ if there exists $k \in \mathbb{Z}$ such that $a_{i}=b_{i+k}$ for all $i$ sufficiently large. Dougherty, 
Jackson and Kechris showed that $E_{t}$ is Borel reducible to $E_{0}$, and so it is hyperfinite [DJK94, Cor 8.2]. It is not hard to see that the orbit equivalence relation induced by the action of $F_{n}$ on $\partial F_{n}$ is Borel reducible to $E_{t}$ with finite $\Omega$. Hence that orbit equivalence relation on $\partial F_{n}$ is hyperfinite, which we will shortly express by saying that the boundary action of $F_{n}$ is hyperfinite.

More recently, Huang, Shinko and Sabok [HSS19] showed that for cocompactly cubulated hyperbolic groups, their boundary actions are hyperfinite. The proof relied on a study of geodesic ray bundles in hyperbolic groups. While Touikan [Tou18] showed that that approach does not work for arbitrary hyperbolic groups, Marquis [Mar19] used it to prove the hyperfiniteness of boundary actions of groups acting cocompactly on locally finite hyperbolic buildings with trivial chamber stabilisers. Very recently, Marquis and Sabok [MS20] showed the hyperfiniteness of the boundary action of an arbitrary hyperbolic group.

\section{Organisation}

In Section 2 we recall the basics on arcs, laminations and unicorn paths. In Section 3 we prove a pair of key lemmas: the local characterisation of Pho-on's infinite unicorn paths and the tail equivalence for asymptotic infinite unicorn paths. This allows for the proofs of Theorem 1.1 and Corollary $1.2 \mathrm{in}$ Section 4. We prove Corollary 1.3 in Section 5.

\section{Unicorn paths}

\subsection{Arcs and laminations}

As in the introduction, $S$ is obtained from a closed oriented surface of genus $g$ by removing $n$ points. Thus $S$ has $n$ topological ends, which are called punctures. An oriented arc on $S$ is a map from $(0,1)$ to $S$ that is proper. A proper map induces a map between topological ends of spaces, and in this sense each endpoint of $(0,1)$ is sent to a puncture of $S$. We will say that the oriented arc starts and ends at these punctures. A homotopy between oriented $\operatorname{arcs} a$ and $b$ is a proper map $(0,1) \times[0,1] \rightarrow S$ whose restriction to $(0,1) \times\{0\}$ equals $a$ and whose restriction to $(0,1) \times\{1\}$ equals $b$. In particular, $a$ and $b$ start at the same puncture and end at the same puncture. A curve on $S$ is a map from a circle $S^{1}$ to $S$.

An oriented arc or a curve is simple if it is an embedding. In that case we can and will identify the oriented arc or the curve with its image in $S$. We record, however, the orientation of the arc, while for the curve we discard it. A curve is essential if it is not homotopically trivial. A curve $c: S^{1} \rightarrow S$ is nonperipheral if it cannot be homotoped into the puncture, in the sense that there is no proper map $S^{1} \times[0,1) \rightarrow S$ whose restriction to $S^{1} \times\{0\}$ is $c$. An oriented arc $a:(0,1) \rightarrow S$ is essential if there is no proper map $(0,1) \times[0,1) \rightarrow S$ whose restriction to $(0,1) \times\{0\}$ is $a$. Unless otherwise stated, all oriented arcs in this paper are simple and essential, and all curves are simple, essential and nonperipheral.

Suppose that the Euler characteristic $\chi=2-2 g-n$ of $S$ is negative. If $n \geq 1$, the $\operatorname{arc} \operatorname{graph} \mathcal{A}(S)$ is the graph whose vertex set $A$ is the set of homotopy classes of oriented arcs on $S$. Two vertices in $A$ are connected by an edge if they can be realised disjointly. Note that since our arcs are oriented, our $\mathcal{A}(S)$ differs from the usual arc graph by replacing each vertex with two.

Allow now $n=0$, but suppose that we are not in one of the exceptional cases where $g=0$ and $n=3$ or 4 , or $g=1$ and $n=1$. Then the curve graph $\mathcal{C}(S)$ is the graph whose vertices are the homotopy classes of curves on $S$. Again, two vertices are connected by an edge if they can be realised disjointly. In the exceptional cases the edges of $\mathrm{C}(S)$ are defined differently, but we will not be appealing to that definition. By [MM99] and [MS13], the graphs $\mathcal{C}(S)$ and $\mathcal{A}(S)$ are Gromov-hyperbolic.

We fix an arbitrary complete hyperbolic metric on $S$. A geodesic lamination on $S$ is a compact subset of $S$ that is a disjoint union of leaves that are geodesic lines and circles in $S$ that do not self-intersect. A geodesic lamination $L$ is minimal if its every leaf is dense in $L$. Let $Y \subseteq S$ be a subsurface whose boundary components are all geodesic circles. We say that a geodesic lamination $L \subset Y$ fills $Y$ if every curve on $Y$ intersects $L$. Analogously, a pair of oriented $\operatorname{arcs} a, b \subset Y$ fills $Y$ if every curve on $Y$ intersects the geodesic representative of $a$ or $b$. 
A peripherally ending lamination is a minimal geodesic lamination that fills a subsurface $Y$ containing all the punctures of $S$. An ending lamination is a minimal geodesic lamination that fills the entire $S$. Let $\mathcal{E} \mathcal{L}(S) \subset \mathcal{E} \mathcal{L}_{0}(S)$ denote the sets of ending and peripherally ending laminations on $S$, respectively, with the topology given by the following coarse Hausdorff convergence. Namely, $L_{n} \stackrel{\mathrm{CH}}{\longrightarrow} L$ if for any subsequence $L_{n_{k}}$ Hausdorff converging to a geodesic lamination $L^{\prime}$, we have $L \subset L^{\prime}$ [Ham06]. By [Kla99] and [Sch13] (see also Theorem 3.2), the spaces $\mathcal{E} \mathcal{L}(S), \mathcal{E} \mathcal{L}_{0}(S)$ can be equivariantly identified with the Gromov boundaries of $\mathcal{C}(S)$ and $\mathcal{A}(S)$.

\subsection{Unicorns}

As in Section 2.1, let $A$ denote the set of homotopy classes of oriented $\operatorname{arcs}$ on $S$.

Definition 2.1. Set $a, b \in A$ and keep the notation $a, b$ for the geodesic oriented arcs representing them. A unicorn arc for $a$ and $b$ is the homotopy class of an oriented arc that is a concatenation $a^{\prime} \cup b^{\prime}$ for $a^{\prime}$ an initial segment of $a$ and $b^{\prime}$ a terminal segment of $b$, possibly $a^{\prime}=a, b^{\prime}=\emptyset$ or $a^{\prime}=\emptyset, b^{\prime}=b$. Note that orienting the arcs replaces the choice of endpoints in [HPW15, Def 3.1].

The set of all oriented arcs that are such concatenations $a^{\prime} \cup b^{\prime}$ can be ordered into a sequence $\left(a_{i}^{\prime} \cup b_{i}^{\prime}\right)_{i=0}^{n}$ so that for all $0 \leq i<n$, we have $a_{i+1}^{\prime} \subset a_{i}^{\prime}$ and $b_{i+1}^{\prime} \supset b_{i}^{\prime}$. We denote by $c_{i} \in A$ the homotopy class of $a_{i}^{\prime} \cup b_{i}^{\prime}$ and we call the sequence $P(a, b)=\left(c_{i}\right)_{i=0}^{n} \in A^{n+1}$ the unicorn path from $a$ to $b$.

Note that we have $c_{0}=a$ and $c_{n}=b$. Moreover, the unicorn path is indeed an edge path in $\mathcal{A}(S)$ :

Remark 2.2 ([HPW15, Rm 3.2]). For each $0 \leq i<n$, the unicorn $\operatorname{arcs} c_{i}, c_{i+1}$ are adjacent in $\mathcal{A}(S)$.

Let $L_{0}$ be a peripherally ending lamination. Let $l$ be a geodesic line on $S$ that does not self-intersect and ends at a puncture in the sense that $l$ contains a geodesic ray properly embedded in $S$. We say that $l$ is asymptotic to $L_{0}$ if $l \subset S \backslash L_{0}$. Since each puncture of $S$ lies in a once-punctured ideal polygon of $S \backslash L_{0}$, the number of such $l$ is bounded by the total number of their ideal vertices, which is at most $2|\chi|$.

Definition 2.3 ([PO17, §3.1]). Set $a \in A$ and keep the notation $a$ for the geodesic oriented arc representing it. Let $l$ be a geodesic line asymptotic to $L_{0} \in \mathcal{E} \mathcal{L}_{0}(S)$. A unicorn arc for $a$ and $l$ is the homotopy class of an oriented arc that is a concatenation $a^{\prime} \cup l^{\prime}$ for $a^{\prime}$ an initial segment of $a$ and $l^{\prime}$ a terminal segment of $l$, possibly $a^{\prime}=a$ and $l^{\prime}=\emptyset$.

The set of all oriented arcs that are such concatenations $a^{\prime} \cup l^{\prime}$ can be ordered into a sequence $\left(a_{i}^{\prime} \cup l_{i}^{\prime}\right)_{i=0}^{\infty}$ so that for all $i \geq 0$, we have $a_{i+1}^{\prime} \subset a_{i}^{\prime}$ and $l_{i+1}^{\prime} \supset l_{i}^{\prime}$. We denote by $c_{i} \in A$ the homotopy class of $a_{i}^{\prime} \cup l_{i}^{\prime}$ and we call the sequence $P(a, l)=\left(c_{i}\right)_{i=0}^{\infty} \in A^{\mathbb{N}}$ the infinite unicorn path from $a$ to $l$.

\section{Key lemmas}

Definition 3.1. Set $n \in\{3,4, \ldots, \infty\}$. A sequence $\left(c_{i}\right)_{i=0}^{n} \in A^{n+1}$ is a locally unicorn path if for each $0 \leq j<k \leq n$, with $j+3 \leq k<\infty$, the sequence $\left(c_{i}\right)_{i=j}^{k}$ is the unicorn path from $c_{j}$ to $c_{k}$.

By Remark 2.2, a locally unicorn path is an edge path in $\mathcal{A}(S)$. Moreover, by [HPW15, Lem 3.5] each finite unicorn path of length $\geq 3$ is a locally unicorn path. Furthermore, by [PO17, Lem 3.4] an infinite unicorn path is also locally unicorn.

By [HPW15, Prop 4.2] there is a universal constant $C$ such that each finite unicorn path $P(a, b)$ is at Hausdorff distance $\leq C$ from a geodesic edge path in $\mathcal{A}(S)$ from $a$ to $b$. Consequently, each locally unicorn path is bounded or converges with respect to the Gromov product [GdlH90, §7.2] to a point in $\partial \mathcal{A}(S)$. This leads to the following result of Pho-on (the existence of an equivariant homeomorphism was announced earlier by Schleimer [Sch13]):

Theorem 3.2 ([PO17, §3.2-3]). Set $a \in A$. Set $L_{0} \in \mathcal{E} \mathcal{L}_{0}(S)$ and let $l$ be a geodesic line asymptotic to $L_{0}$. Then $P(a, l)$ is not bounded and its limit $F\left(L_{0}\right) \in \partial \mathcal{A}(S)$ with respect to the Gromov product depends only on $L_{0}$. Furthermore, $F: \mathcal{E} \mathcal{L}_{0}(S) \rightarrow \partial \mathcal{A}(S)$ is a $\operatorname{Mod}(S)$-equivariant homeomorphism. 


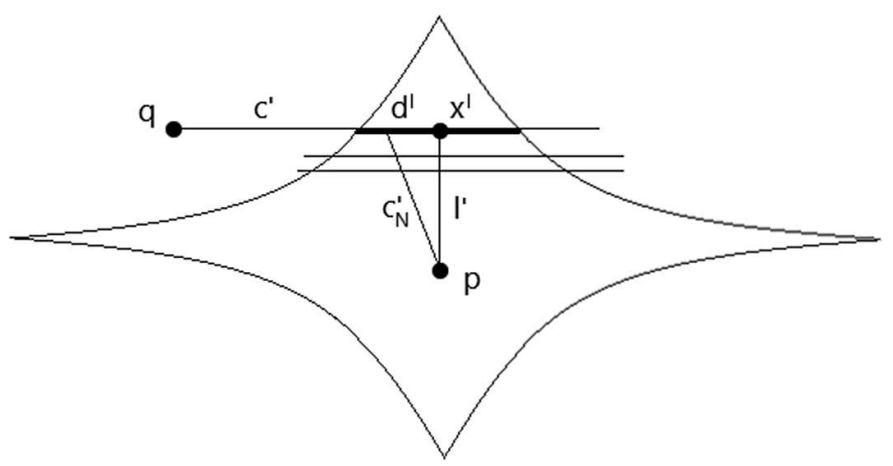

Figure 1. Ideal polygon D.

In fact, the local condition characterises infinite unicorn paths:

Lemma 3.3. Let $P$ be a locally unicorn path that is not bounded in $\mathcal{A}(S)$. Then $P$ is an infinite unicorn path.

Proof. Denote $P=\left(c_{i}\right)_{i=0}^{\infty} \in A^{\mathbb{N}}$, and keep the notation $c_{i}$ for the geodesic oriented arcs representing them. Since $P$ is not bounded in $\mathcal{A}(S)$, it converges to some point $F\left(L_{0}\right) \in \partial \mathcal{A}(S)$. By [PO17, Lem 3.9], we have that $c_{i}$ coarse Hausdorff converge to $L_{0} \in \mathcal{E} \mathcal{L}_{0}(S)$. Denote $c=c_{0}$. We claim that for each $n \geq 1$ there is a geodesic line $l$ asymptotic to $L_{0}$ such that for each $i \leq n$, the unicorn arc $a_{i}$ on the infinite unicorn path from $c$ to $l$ coincides with $c_{i}$. Since there are only finitely many $l$ asymptotic to $L_{0}$, the lemma follows from the claim.

To justify the claim, note that since $P$ is a locally unicorn path, all $c_{i}$ with $i \geq 1$ end at a common puncture $p$. Let $D$ be the ideal polygon of $S \backslash L_{0}$ containing $p$. Let $l$ be a geodesic line asymptotic to $L_{0}$ ending at $p$. Let $c^{\prime} \cup l^{\prime}$ represent the $n$th unicorn arc on the unicorn path from $c$ to $l$, let $x^{l}=c^{\prime} \cap l^{\prime}$ and let $d^{l}$ be the segment of $c$ that is the component of $D \cap c$ containing $x^{l}$ (see Figure 1). Let $D_{p}$ be the component of $D-\bigcup_{l} d^{l}$ containing $p$, where the union is taken over all the geodesic lines $l$ asymptotic to $L_{0}$ and ending at $p$. Let $\alpha>0$ be the minimum possible angle that makes with $L_{0}$ a geodesic ray in $D_{p}$ starting on $L_{0}$ and ending at $p$. Since $\left(c_{i}\right)_{i=0}^{\infty} \stackrel{\mathrm{CH}}{\longrightarrow} L_{0}$, there is $N \geq n$ such that $c_{N}$ does not intersect $L_{0}$ at angle $\geq \alpha$. Consequently, the component $c_{N}^{\prime}$ of $c_{N} \cap D_{p}$ ending at $p$ starts on $d^{l}$ for some $l$ (see Figure 1).

Let $q$ be the puncture at which $c$ starts. We have a bijection $h: l^{\prime} \cap c \rightarrow c_{N}^{\prime} \cap c$ such that each pair $x, h(x)$ lies in the same component of $D_{p} \cap c$. Furthermore, for each $x \in l^{\prime} \cap c$, the segments $q x \subset c$ and $x p \subset l$ intersect only at $x$ if and only if the segments $q h(x) \subset c$ and $h(x) p \subset c_{N}$ intersect only at $h(x)$. In other words, the concatenation $q x \cup x p$ represents a unicorn arc for $c$ and $l$ if and only if the concatenation $q h(x) \cup h(x) p$ represents a unicorn arc for $c$ and $c_{N}$. Moreover, these two oriented arcs are homotopic. Finally, this correspondence preserves the order of unicorn arcs. Thus, for $0 \leq i \leq n$, we have $a_{i}=c_{i}$, justifying the claim.

Set $L_{0} \in \mathcal{E} \mathcal{L}_{0}(S)$. We define an equivalence relation $\sim_{L_{0}}$ on $A$ by declaring $a \sim_{L_{0}} b$ if the geodesic representatives of $a, b$ start at the same puncture and their first points in $L_{0}$ lie on the same side of the ideal polygon of $S \backslash L_{0}$ containing that puncture. Note that $\sim_{0}$ has at most $2|\chi|$ equivalence classes.

We now prove a tail equivalence lemma that will later allow us to reduce the orbit equivalence on $\partial \mathcal{A}(S)$ to $E_{t}$.

Lemma 3.4. Set $L_{0} \in \mathcal{E} \mathcal{L}_{0}(S)$, and set $a, b \in A$ with $a \sim{ }_{L_{0}} b$. Then for each geodesic line $l$ asymptotic to $L_{0}$, the unicorn path $\left(a_{i}\right)_{i=0}^{\infty}$ from a to $l$ and the unicorn path $\left(b_{i}\right)_{i=0}^{\infty}$ from $b$ to $l$ satisfy $a_{i}=b_{i+k}$ for some $k \in \mathbb{Z}$ and all $i$ sufficiently large. 


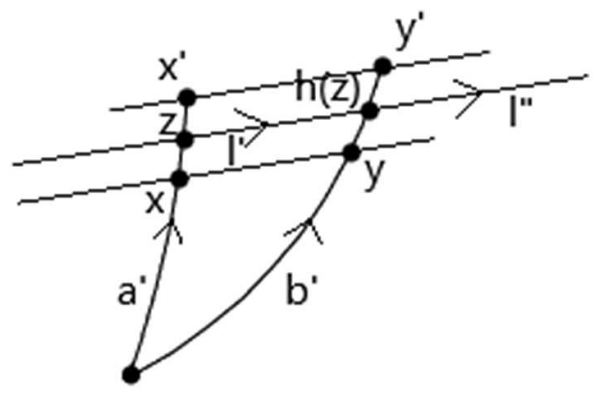

Figure 2. Rectangle B.

Proof. Set $a, b \in A$ with $a \sim_{L_{0}} b$ and keep the notation $a, b$ for the geodesic oriented arcs representing them. Let $x, y$ be the first points on $a, b$ in $L_{0}$. Since $a \sim L_{0} b$, there is a geodesic segment $x y \subset L_{0}$. Furthermore, since $L_{0}$ is minimal, there are segments $x x^{\prime}, y y^{\prime}$ in $a, b$ such that $x^{\prime} y^{\prime}$ is a geodesic segment in $L_{0}$ and $x x^{\prime} y^{\prime} y$ bounds a topological disc $B$ embedded in $S$ (see Figure 2).

Consequently, the components of the intersection $B \cap l$ are geodesic segments joining $x x^{\prime}$ to $y y^{\prime}$, which yields a bijection $h: x x^{\prime} \cap l \rightarrow y y^{\prime} \cap l$. Let $a^{\prime} \subset a$ be an initial segment of $a$ ending in $z \in x x^{\prime} \cap l$, and let $b^{\prime}$ be the initial segment of $b$ ending in $h(z)$. Furthermore, let $l^{\prime}, l^{\prime \prime}$ be the terminal segments of $l$ starting in $z, h(z)$, respectively. Assume without loss of generality $l^{\prime \prime} \subset l^{\prime}$, as in Figure 2.

Note that $x z$ intersects $z h(z) \subset l^{\prime}$ only at $z$. Furthermore, $x z$ is disjoint from $l^{\prime \prime}$ if and only if $y h(z)$ intersects $l^{\prime \prime}$ only at $h(z)$. Consequently, the concatenation $a^{\prime} \cup l^{\prime}$ represents a unicorn arc if and only if $b^{\prime} \cup l^{\prime \prime}$ represents a unicorn arc. Moreover, these two oriented arcs are homotopic. Finally, this correspondence preserves the order of unicorn arcs, and all $a_{i}$ for $i$ sufficiently large are accounted for in this way.

\section{Hyperfiniteness}

We fix a base point $a_{0} \in A$.

Definition 4.1. Let $\mathcal{P} \subset A^{\mathbb{N}}$ be the set of infinite unicorn paths from $a_{0}$ to any geodesic line asymptotic to any $L_{0} \in \mathcal{E} \mathcal{L}_{0}(S)$. Let $f: \mathcal{P} \rightarrow \partial \mathcal{A}(S)$ be the map assigning to such path its limit $F\left(L_{0}\right) \in \partial \mathcal{A}(S)$ with respect to the Gromov product (see Theorem 3.2).

Note that $f$ is finite-to-one, since $a_{0}$ is fixed and there are finitely many geodesic lines asymptotic to a given $L_{0} \in \mathcal{E} \mathcal{L}_{0}(S)$.

Remark 4.2. We equip the countable set $A$ with the discrete topology and $A^{\mathbb{N}}$ with the product topology. Then the set $\mathcal{P} \subset A^{\mathbb{N}}$ is Borel. Indeed, by Lemma 3.3, $\mathcal{P}$ is the set of locally unicorn paths that are not bounded. The set of locally unicorn paths is closed in $A^{\mathbb{N}}$, since each of the conditions on $\left(c_{i}\right)_{i=j}^{k}$ to be a unicorn path is closed. Furthermore, for each $n \geq 0$, the set of sequences in $A^{\mathbb{N}}$ at distance $\leq n$ from $a_{0}$ is closed, so the set of sequences in $A^{\mathbb{N}}$ at bounded distance from $a_{0}$ is a countable union of closed sets. Consequently, $\mathcal{P}$ is a countable intersection of open sets.

Since locally unicorn paths are uniformly Hausdorff close to geodesic edge paths, and the function $f$ assigns their limits in $\partial \mathcal{A}(S)$, we have that $f$ is continuous with respect to the metric on $\partial \mathcal{A}(S)$ defined using the Gromov product.

Let $\mathcal{T}$ be the countable set of finite-length edge paths in $\mathcal{A}(S)$, up to the action of $\operatorname{Mod}(S)$, equipped with the discrete topology. For an infinite unicorn path $P=\left(c_{i}\right)_{i=0}^{\infty}$, given $i \geq 0$ and $j=i+1, \ldots$, the subsurfaces $\Sigma_{i, j} \subseteq S$ filled by $c_{i}$ and $c_{j}$ form an ascending sequence $\Sigma_{i, i+1} \subseteq \Sigma_{i, i+2} \subseteq \cdots$ that stabilises with some subsurface, which we call $\Sigma_{i} \subseteq S$. For each $i \geq 0$, let $m(i)>i+1$ be minimal satisfying $\Sigma_{i+1, m(i)}=\Sigma_{i+1}$. Let $T_{i}=\left(c_{j}\right)_{j=i}^{m(i)}$, and let $\left[T_{i}\right]$ be the equivalence class of $T_{i}$ in $\mathcal{T}$. Let $g: \mathcal{P} \rightarrow \mathcal{T}^{\mathbb{N}}$ be 
the map defined by $g(P)=\left(\left[T_{i}\right]\right)_{i=0}^{\infty}$. Let $E_{t}$ be the tail equivalence relation on $\mathcal{T}^{\mathbb{N}}$ described in Section 1 (with $\Omega=\mathcal{T}$ ).

Note that the definition of $g$ can be analogously extended to infinite unicorn paths $P \notin \mathcal{P}$ (that is, to infinite unicorn paths that start at points distinct from $a_{0}$ ), which we will make use of later on.

Remark 4.3. We equip $\mathcal{T}^{\mathbb{N}}$ with the product topology. Then the map $g: \mathcal{P} \rightarrow \mathcal{T}^{\mathbb{N}}$ is Borel. Indeed, for all $0 \leq i<j$, the maps $P \rightarrow \Sigma_{i, j}$ are continuous maps from $\mathcal{P}$ to the countable discrete set of subsurfaces of $S$, and hence their limits $P \rightarrow \Sigma_{i}$ are Borel. Thus, for all $0 \leq i<j$, the subset of $\mathcal{P}$ defined by the identity $\Sigma_{i, j}=\Sigma_{i}$ is Borel, and so the maps $m(i): \mathcal{P} \rightarrow \mathbb{N}$ are Borel. Consequently, all the maps $\left[T_{i}\right]: \mathcal{P} \rightarrow \mathcal{T}$ are Borel, as desired.

Lemma 4.4. Set $P, P^{\prime} \in \mathcal{P}$. If $g(P) \sim_{E_{t}} g\left(P^{\prime}\right)$, then there is $\psi \in \operatorname{Mod}(S)$ satisfying $\psi f(P)=f\left(P^{\prime}\right)$. Conversely, for each orbit $\omega$ of the action of $\operatorname{Mod}(S)$ on $\partial \mathcal{A}(S)$, there are finitely many equivalence classes of $E_{t}$ on $\mathcal{T}^{\mathbb{N}}$ containing all $g(P)$ for $P \in \mathcal{P}$ with $f(P) \in \omega$.

Proof. Denote $P=\left(c_{i}\right)_{i=0}^{\infty}, P^{\prime}=\left(c_{i}^{\prime}\right)_{i=0}^{\infty}$. Let $T_{i}^{\prime}$ be defined for $P^{\prime}$ analogously as $T_{i}$ was for $P$. If $g(P) \sim_{E_{t}} g\left(P^{\prime}\right)$, then there are $k \in \mathbb{Z}, j \in \mathbb{N}$ such that $\left[T_{i}\right]=\left[T_{i+k}^{\prime}\right]$ for all $i \geq j$. In particular, there is $\psi \in \operatorname{Mod}(S)$ with $\psi T_{j}=T_{j+k}^{\prime}$. We will show inductively that $\psi T_{i}=T_{i+k}^{\prime}$ for all $i \geq j$, so in particular $\psi c_{i}=c_{i+k}^{\prime}$ implying $\psi f(P)=f\left(P^{\prime}\right)$.

Suppose that we have established $\psi T_{i}=T_{i+k}^{\prime}$ for some $i \geq j$. If $m(i+1) \leq m(i)$, then $\psi T_{i+1}=T_{i+1+k}^{\prime}$ is immediate, so we can assume $m(i+1)>m(i)$. Let $\rho \in \operatorname{Mod}(S)$ be such that $\rho T_{i+1}=T_{i+1+k}^{\prime}$. Then $\rho^{-1} \psi$ fixes all $c_{i+1}, \ldots, c_{m(i)}$. Thus the restriction of $\rho^{-1} \psi$ to the subsurface $\Sigma_{i+1} \subset S$, which $c_{i+1}$ and $c_{m(i)}$ fill, is the identity map. By the definition of $\Sigma_{i+1}$, we have that $c_{m(i)+1}, \ldots, c_{m(i+1)}$ all lie in $\Sigma_{i+1}$. This implies that $\rho^{-1} \psi$ fixes them, and so $\psi T_{i+1}=T_{i+1+k}^{\prime}$, completing the induction.

For the converse, let $\omega$ be the orbit under $\operatorname{Mod}(S)$ of some $F\left(L_{0}\right) \in \partial \mathcal{A}(S)$. Let $l_{1}, \ldots, l_{n}$ be the finitely many geodesic lines asymptotic to $L_{0}$. Choose $a_{1}, \ldots, a_{p} \in A$ that are representatives of the equivalence classes of $\sim_{L_{0}}$ distinct from the one containing $a_{0}$. For $0 \leq q \leq p$ and $1 \leq j \leq n$, let $P_{q j}=P\left(a_{q}, l_{j}\right)$.

Set $P=\left(c_{i}\right)_{i=0}^{\infty} \in \mathcal{P}$ with $f(P) \in \omega$. By Theorem 3.2, we have $P=P\left(a_{0}, l\right)$, where $l$ is a geodesic line asymptotic to $\psi L_{0}$ for some $\psi \in \operatorname{Mod}(S)$. In particular, for some $1 \leq j \leq n$ we have $l=\psi l_{j}$. Thus $\psi^{-1} P=P\left(\psi^{-1} a_{0}, l_{j}\right)$. Choose $0 \leq q \leq p$ so that $a_{q} \sim_{L_{0}} \psi^{-1} a_{0}$. By Lemma 3.4, writing $P_{q j}=\left(b_{i}\right)_{i=0}^{\infty}$, we have $\psi^{-1} c_{i}=b_{i+k}$ for some $k \in \mathbb{Z}$ and all $i$ sufficiently large. We have then that $g\left(P_{q j}\right)$ and $g\left(\psi^{-1} P\right)$, hence also $g(P)$, are tail equivalent.

Proof of Theorem 1.1. Write $E$ for the equivalence relation on $\partial \mathcal{A}(S)$ induced by the action of $\operatorname{Mod}(S)$ and write $E^{*}$ for the equivalence relation on $\mathcal{P}$ that is the pullback of $E$ via $f$ - that is, $P \sim_{E^{*}} P^{\prime}$ if $f(P) \sim_{E} f\left(P^{\prime}\right)$. Since $E$ is Borel and countable, and $f$ is Borel and finite-to-one, we have that $E^{*}$ is also Borel and countable.

Since $f$ is a Borel finite-to-one function, it has a Borel right inverse by the Lusin-Novikov uniformisation theorem [Kec95, Thm 18.10]. Consequently, $E$ is Borel reducible to $E^{*}$. Thus it is enough to show that $E^{*}$ is hyperfinite.

Write $E_{t}^{*}$ for the equivalence relation on $\mathcal{P}$ that is the pullback of $E_{t}$ via $g$. Since $E_{t}$ is Borel, and $g$ is Borel, we have that $E_{t}^{*}$ is Borel. By Lemma 4.4, we have $E_{t}^{*} \subseteq E^{*}$ and every equivalence class of $E^{*}$ contains finitely many equivalence classes of $E_{t}^{*}$. (In particular, $E_{t}^{*}$ is countable.) Thus by [JKL02, Prop 1.3(vii)], it is enough to show that $E_{t}^{*}$ is hyperfinite.

Note that $g$ is a Borel reduction of $E_{t}^{*}$ to $E_{t}$. Thus since $E_{t}$ is hyperfinite [DJK94, Cor 8.2], we have that $E_{t}^{*}$ is hyperfinite as well.

Proof of Corollary 1.2. Assume first that $S$ has $n \geq 1$ punctures. Then by [Kla99, Thm 1.3], Theorem 1.1 and [JKL02, Prop 1.3(iii)], it suffices to prove that $\mathcal{E} \mathcal{L}(S)$ is a Borel subset of $\mathcal{E} \mathcal{L}_{0}(S)$. Indeed, $L_{0} \in$ $\mathcal{E} \mathcal{L}_{0}(S)$ is a minimal filling lamination if and only if each geodesic representative of a curve $c$ on $S$ intersects $L_{0}$ and does it transversally. Given $c$, this is an open condition, and so $\mathcal{L} \mathcal{L}(S)$ is a countable intersection of open sets. 
Second, assume $n=0$ and let $S^{\prime}$ be the surface obtained from $S$ by adding one puncture at a point outside the closure of the union of all embedded geodesic circles and lines, which exists by [BS85, Thm I]. This induces a closed embedding $e: \mathcal{E} \mathcal{L}(S) \rightarrow \mathcal{E} \mathcal{L}\left(S^{\prime}\right)$, which is a section for the map $r: \mathcal{E} \mathcal{L}\left(S^{\prime}\right) \rightarrow \mathcal{E} \mathcal{L}(S)$ defined by forgetting the puncture (see [PO17, §4.2] for details). Thus for each $L_{1}, L_{2} \in \mathcal{E} \mathcal{L}(S)$, with $\psi^{\prime} e\left(L_{1}\right)=e\left(L_{2}\right)$ for some $\psi^{\prime} \in \operatorname{Mod}\left(S^{\prime}\right)$, the image $\psi \in \operatorname{Mod}(S)$ of $\psi^{\prime}$ under the puncture-forgetting $\operatorname{map} \operatorname{Mod}\left(S^{\prime}\right) \rightarrow \operatorname{Mod}(S)$ satisfies $\psi\left(L_{1}\right)=L_{2}$.

Conversely, set $L \in \mathcal{E} \mathcal{L}(S)$ and let $R_{1}, \ldots, R_{n} \subset S$ be the components of $S \backslash L$. For $1 \leq j \leq n$, let $L_{j}$ be a lamination in $\mathcal{E} \mathcal{L}\left(S^{\prime}\right)$ obtained from $L$ by adding a puncture in $R_{j}$, under an arbitrary identification with $S^{\prime}$. All such identifications differ by $\operatorname{Mod}\left(S^{\prime}\right)$, so the resulting orbit $\left[L_{j}\right]$ in $\mathcal{E} \mathcal{L}\left(S^{\prime}\right)$ does not depend on our choice. Since $e$ is a section for $r$, we have $e(L) \in \bigcup_{j=1}^{n}\left[L_{j}\right]$. Analogously, for any $\psi \in \operatorname{Mod}(S)$, we have $e(\psi(L)) \in \bigcup_{j=1}^{n}\left[L_{j}\right]$.

Consequently, under the identification of $\mathcal{E} \mathcal{L}(S)$ with $e(\mathcal{E} \mathcal{L}(S))$, each orbit of $\operatorname{Mod}(S)$ on $\mathcal{E} \mathcal{L}(S)$ consists of the intersections of finitely many orbits of $\operatorname{Mod}\left(S^{\prime}\right)$ on $\mathcal{E} \mathcal{L}\left(S^{\prime}\right)$ with $e(\mathcal{E} \mathcal{L}(S))$. Thus by [JKL02, Prop 1.3 (iii,vii)], the hyperfiniteness of the action of $\operatorname{Mod}(S)$ on $\mathcal{E} \mathcal{L}(S)$ follows from the hyperfiniteness of the action of $\operatorname{Mod}\left(S^{\prime}\right)$ on $\mathcal{E} \mathcal{L}\left(S^{\prime}\right)$.

\section{Complete geodesic laminations}

A geodesic lamination $L$ on $S$ is complete if each component of $S \backslash L$ is an ideal triangle or a oncepunctured monogon, and $L$ lies in the closure (in the Hausdorff topology on the space of compact subsets of $S$ ) of the set of embedded geodesic circles. By $\mathcal{C} L(S)$ we denote the space of complete geodesic laminations with the Hausdorff topology, which is compact and Hausdorff [Ham09, §2.1].

Proof of Corollary 1.3. For a complete geodesic lamination $L$, let $L^{\prime}$ denote the union of minimal sublaminations of $L$ that are not embedded geodesic circles. Let $Y(L)$ denote the subsurface of $S$ filled by $L^{\prime}$. Given a subsurface $Y \subseteq S$, let $\mathcal{C} L(S, Y) \subset \mathcal{C} L(S)$ denote the subspace of laminations $L$ with $Y(L)=Y$. We claim that each $\mathcal{C} L(S, Y)$ is a Borel subset of $\mathcal{C} L(S)$, and hence $\operatorname{Mod}(S) \mathcal{C} L(S, Y)$ is Borel as well.

Indeed, for a lamination $L$ in $\mathcal{C} L(S, Y)$, the union $Z$ of the nonisolated leaves of $L$ on $S \backslash Y$ is a union of disjoint (geodesic representatives of) curves on $S \backslash Y$. Thus a complete geodesic lamination $L$ belongs to $\mathrm{C} L(S, Y)$ if and only if

- each curve $c$ on $Y$ intersects $L$ transversally an infinite number of times ( $G_{\delta}$ condition) and

$\circ$ there exists a union of disjoint curves $Z$ on $S \backslash Y$ such that each curve $c$ on $S \backslash(Y \cup Z)$ or in $Z \cup \partial Y$ does not intersect $L$ transversally an infinite number of times ( $F_{\sigma \delta \sigma}$ condition).

Thus $\mathrm{C} L(S, Y)$ is an $F_{\sigma \delta \sigma}$ set, justifying the claim.

By [JKL02, Prop 1.3(v)], to prove that the orbit equivalence relation on $\mathrm{C} L(S)$ induced by the action on $\operatorname{Mod}(S)$ is hyperfinite, it suffices to show that its restriction to each $\operatorname{Mod}(S) \mathcal{C} L(S, Y)$ is hyperfinite. By [JKL02, Prop 1.3(vi)], it suffices to show that the orbit equivalence relation $E$ on $\mathcal{C} L(S, Y)$ induced by the action of the stabiliser $\operatorname{Mod}(S)_{Y}$ of $Y$ in $\operatorname{Mod}(S)$ is hyperfinite.

Let $Y_{1}, \ldots, Y_{k}$ be the components of $Y$, where we treat all geodesic boundary components as punctures. Let $g: \mathcal{C} L(S, Y) \rightarrow \mathcal{E} \mathcal{L}\left(Y_{1}\right) \times \cdots \times \mathcal{E} \mathcal{L}\left(Y_{k}\right)$ be the map assigning to each $L$ the components of its sublamination $L^{\prime}$. In the case where $Y=\emptyset$, the product $\mathcal{E} \mathcal{L}\left(Y_{1}\right) \times \cdots \times \mathcal{E} \mathcal{L}\left(Y_{k}\right)$ should be understood as a point. By Corollary 1.2 and [JKL02, Prop 1.3(iv)], the orbit equivalence relation on $\mathcal{E} \mathcal{L}\left(Y_{1}\right) \times \cdots \times \mathcal{E} \mathcal{L}\left(Y_{k}\right)$ induced by the action of $\operatorname{Mod}\left(Y_{1}\right) \times \cdots \times \operatorname{Mod}\left(Y_{k}\right)$ is hyperfinite. The group $\operatorname{Mod}\left(Y_{1}\right) \times \cdots \times \operatorname{Mod}\left(Y_{k}\right)$ is of finite index in $\operatorname{Mod}\left(Y_{1} \sqcup \cdots \sqcup Y_{k}\right)$. Thus by [JKL02, Prop 1.3(vii)], the orbit equivalence relation on $\mathcal{E} \mathcal{L}\left(Y_{1}\right) \times \cdots \times \mathcal{E} \mathcal{L}\left(Y_{k}\right)$ induced by the action of $\operatorname{Mod}\left(Y_{1} \sqcup \cdots \sqcup Y_{k}\right)$ is hyperfinite. Its pullback $F$ under $g$ is thus hyperfinite as well, since $g$ has countable fibres. Since $E$ is contained in $F$, it is hyperfinite by [JKL02, Prop 1.3(i)], as desired. 
Acknowledgements. We thank Camille Horbez for the input on the $\operatorname{Out}\left(F_{n}\right)$ case, Jean Renault for helpful explanations and the referee for valuable remarks. We also thank Antoni Sabok-Przytycki for encouragement. The first author was partially supported by AMS, and both authors were partially supported by NSERC and the National Science Centre, Poland UMO-2018/30/M/ST1/00668.

\section{References}

[Ada94] S. Adams, 'Boundary amenability for word hyperbolic groups and an application to smooth dynamics of simple groups', Topology 33(4) (1994), 765-783.

[AEG94] S. Adams, G. A. Elliott and T. Giordano, 'Amenable actions of groups', Trans. Amer. Math. Soc. 344(2) (1994), 803-822.

[AD87] C. Anantharaman-Delaroche, 'Systèmes dynamiques non commutatifs et moyennabilité', Math. Ann. 279(2) (1987), 297-315.

[AD02] C. Anantharaman-Delaroche, 'Amenability and exactness for dynamical systems and their $C^{*}$-algebras', Trans. Amer. Math. Soc. 354(10) (2002), 4153-4178.

[ADR00] C. Anantharaman-Delaroche and J. Renault, Amenable Groupoids, Monographies de L'Enseignement Mathématique, vol. 36 (L'Enseignement Mathématique, Geneva, 2000). With a foreword by G. Skandalis and Appendix B by E. Germain.

[BGH21] M. Bestvina, V. Guirardel and C. Horbez, 'Boundary amenability of Out $\left(F_{N}\right)$ ', 'Ann. Sci. Éc. Norm. Supér. (4), to appear. Preprint, 2021, arXiv:1705.07017.

[BR15] M. Bestvina and P. Reynolds, 'The boundary of the complex of free factors', Duke Math. J. 164(11) (2015), 2213-2251.

[BS85] J. S. Birman and C. Series, 'Geodesics with bounded intersection number on surfaces are sparsely distributed', Topology 24(2) (1985), 217-225.

[CFW81] A. Connes, J. Feldman and B. Weiss, 'An amenable equivalence relation is generated by a single transformation', Ergodic Theory Dynam. Systems 1(4) (1981), 431-450.

[DJK94] R. Dougherty, S. Jackson and A. S. Kechris, 'The structure of hyperfinite Borel equivalence relations', Trans. Amer. Math. Soc. 341(1) (1994), 193-225.

[Gao09] S. Gao, Invariant Descriptive Set Theory, Pure and Applied Mathematics (Boca Raton), vol. 293 (CRC Press, Boca Raton, FL, 2009).

[GdlH90] É. Ghys and P. de la Harpe (eds.), Sur les groupes hyperboliques d'aprés Mikhael Gromov, Progress in Mathematics, vol. 83 (Birkhäuser Boston, Boston, MA, 1990). Papers from the Swiss Seminar on Hyperbolic Groups held in Bern, 1988.

[GHL20] V. Guirardel, C. Horbez and J. Lécureux, 'Cocycle superrigidity from higher rank lattices to Out $\left(F_{N}\right)$ ', Preprint, 2020, arXiv:2005.07477.

[Ham06] U. Hamenstädt, 'Train tracks and the Gromov boundary of the complex of curves', in Spaces of Kleinian Groups, London Math. Soc. Lecture Note Ser., vol. 329 (Cambridge University Press, Cambridge, UK, 2006), 187-207.

[Ham09] U. Hamenstädt, 'Geometry of the mapping class groups, I: Boundary amenability', Invent. Math. 175(3) (2009), 545-609.

[Ham14] U. Hamenstädt, 'The boundary of the free factor graph and the free splitting graph', Preprint, 2014, arXiv:1211.1630.

[HPW15] S. Hensel, P. Przytycki and R. C. H. Webb, '1-slim triangles and uniform hyperbolicity for arc graphs and curve graphs', J. Eur. Math. Soc. (JEMS) 17(4) (2015), 755-762.

[HSS19] J. Huang, F. Shinko and M. Sabok, 'Hyperfiniteness of boundary actions of cubulated hyperbolic groups', Ergodic Theory Dynam. Systems 40(9) (2020), 2453-2466.

[JKL02] S. Jackson, A. S. Kechris and A. Louveau, 'Countable Borel equivalence relations', J. Math. Log. 2(1) (2002), 1-80.

[Kai04] V. A. Kaimanovich, 'Boundary amenability of hyperbolic spaces', in Discrete Geometric Analysis, Contemp. Math., vol. 347 (American Mathematical Society, Providence, RI, 2004), 83-111.

[Kec93] A. S. Kechris, 'Amenable versus hyperfinite Borel equivalence relations', J. Symb. Log. 58(3) (1993), 894-907.

[Kec95] A. S. Kechris, Classical Descriptive Set Theory, Graduate Texts in Mathematics, vol. 156 (Springer-Verlag, New York, 1995).

[KM04] A. S. Kechris and B. D. Miller, Topics in Orbit Equivalence, Lecture Notes in Mathematics, vol. 1852 (SpringerVerlag, Berlin, 2004).

[Kid08] Y. Kida, The Mapping Class Group from the Viewpoint of Measure Equivalence Theory, Mem. Amer. Math. Soc., vol. 196(916) (2008), viii+190.

[Kla99] E. Klarreich, 'The boundary at infinity of the curve complex and the Relative Teichmüller Space', Preprint, 1999, arXiv:1803.10339.

[Léc10] J. Lécureux, 'Amenability of actions on the boundary of a building', Int. Math. Res. Not. IMRN 17 (2010), 3265-3302.

[Mar19] T. Marquis, 'On geodesic ray bundles in buildings', Geom. Dedicata 202 (2019), 27-43.

[MS20] T. Marquis and M. Sabok, 'Hyperfiniteness of boundary actions of hyperbolic groups', Math. Ann. 377 (2020), $1129-1153$. 
[MM99] H. A. Masur and Y. N. Minsky, 'Geometry of the complex of curves, I: Hyperbolicity', Invent. Math. 138(1) (1999), 103-149.

[MS13] H. A. Masur and S. Schleimer, 'The geometry of the disk complex', J. Amer. Math. Soc. 26(1) (2013), 1-62.

[Moo20] J. T. Moore, 'A brief introduction to amenable equivalence relations', in Trends in Set Theory, Contemp. Math., vol. 752 (American Mathematical Society, Providence, RI, 2020), 153-163.

[NS13] A. Nevo and M. Sageev, 'The Poisson boundary of CAT(0)cube complex groups', Groups Geom. Dyn. 7(3) (2013), 653-695.

[Oza06a] N. Ozawa, 'Boundary amenability of relatively hyperbolic groups', Topology Appl. 153(14) (2006), 2624-2630.

[Oza06b] N. Ozawa, Amenable Actions and Applications, International Congress of Mathematicians, vol. II (European Mathematical Society, Zürich, 2006), 1563-1580.

[PO17] W. Pho-on, 'Infinite unicorn paths and Gromov boundaries', Groups Geom. Dyn. 11(1) (2017), 353-370.

[Sch13] S. Schleimer (2013), unpublished manuscript.

[Tou18] N. Touikan, 'On geodesic ray bundles in hyperbolic groups', Proc. Amer. Math. Soc. 146(10) (2018), 4165-4173.

[Ver78] A. M. Veršik, 'The action of PSL (2, Z) in R ${ }^{1}$ is approximable', Uspekhi Mat. Nauk 33(1(199)) (1978), 209-210.

[Zim78] R. J. Zimmer, 'Amenable ergodic group actions and an application to Poisson boundaries of random walks', J. Funct. Anal. 27(3) (1978), 350-372.

[Zim84] R. J. Zimmer, Ergodic Theory and Semisimple Groups, Monographs in Mathematics, vol. 81 (Birkhäuser Verlag, Basel, 1984). 